\title{
Extensive $A R M C 5$ genetic variance in primary bilateral macronodular adrenal hyperplasia that started with exophthalmos: a case report
}

\author{
Ping Jin ${ }^{1}$, Muhammad Usman Janjua' ${ }^{1}$, Qin Zhang ${ }^{1}$, Chang-sheng Dong ${ }^{2}$, Youbo Yang ${ }^{1}$ and Zhao-hui Mo ${ }^{\text {* }}$
}

\begin{abstract}
Background: Primary bilateral macronodular adrenal hyperplasia is a rare cause of Cushing's syndrome characterized by the presence of bilateral secretory adrenal nodules. Recent studies have shown that primary bilateral macronodular adrenal hyperplasia is caused by combined germline and somatic mutations of the ARMC5 gene. Exophthalmos is an underappreciated sign of Cushing's syndrome.

Case presentation: A 52-year-old Chinese woman with progressively worsening bilateral proptosis presented to our hospital. Subsequently she was diagnosed as having primary bilateral macronodular adrenal hyperplasia and underwent bilateral laparoscopic adrenalectomy. Genomic deoxyribonucleic acid was isolated from lymphocytes as well as seven different adrenal nodules and the ARMC5 sequence was determined by Sanger sequencing. We identified one heterozygous ARMC5 germline mutation c.682C>T (p. Gln228*) and five heterozygous somatic mutations (c.310delG, c.347_357del11, c.267delC, c.283_289del7, and c.205-322del118) in five different adrenal nodules. All mutations are novel and were not found in any of the available online databases. To test whether the ARMC5 mutation induced messenger ribonucleic acid decay, real-time reverse transcriptase polymerase chain reaction was performed on patient and control adrenal tissue. We found that the adrenal cortex of our patient showed a Iow ARMC5 messenger ribonucleic acid expression compared with normal adrenal cortex, possibly as a result of nonsense-mediated messenger ribonucleic acid decay
\end{abstract}

Conclusions: We demonstrated extensive genetic diversity of ARMC5 in a patient with primary bilateral macronodular adrenal hyperplasia that started with exophthalmos, which contributes to further understanding of the pathogenesis of this disease. Early recognition of atypical symptoms and screening for ARMC5 mutation in patients with primary bilateral macronodular adrenal hyperplasia has important clinical implications for the diagnosis and genetic counseling.

Keywords: PBMAH, Cushing's syndrome, ARMC5, Exophthalmos

\section{Background}

Primary bilateral macronodular adrenal hyperplasia (PBMAH), also known as adrenocorticotropic hormone (ACTH)-independent macronodular adrenal hyperplasia (AIMAH), is a rare cause of Cushing's syndrome [1, 2], characterized by increased cortisol production from bilateral adrenal macronodules. Recently, the inactivating

\footnotetext{
* Correspondence: ping.jin06@gmail.com

'Department of Endocrinology, The third Xiangya Hospital, Central South University, Tongzipo Road, Changsha 410007, Hunan, People's Republic of China

Full list of author information is available at the end of the article
}

mutations of the armadillo repeat containing 5 (ARMC5, OMIM 615549) gene located at chromosome 16p11.2 were identified as a genetic cause of PBMAH $[3,4]$. This gene has been proposed to acts as a tumor-suppressor gene where patients with PBMAH have a single germline mutation in ARMC5, acting as the first "hit," and subsequent somatic mutation of the gene within adrenal tissue leading to the development of nodules and overproduction of cortisol [5-8]. In the current study, we present a case of a 52-year-old woman who presented to us with bilateral proptosis and was subsequently 
diagnosed as having PBMAH. ARMC5 variations were analyzed in the deoxyribonucleic acid (DNA) of the lymphocytes and adrenal nodules of this patient.

This case report uncovers extensive genetic diversity of $A R M C 5$, mutations which were not found in the available online database, and this case also highlights the importance of atypical symptoms of Cushing's syndrome, such as exophthalmos, the early detection of which can prevent the patient from many detrimental effects of excessive cortisol exposure.

\section{Case presentation}

A 52-year-old Chinese woman with progressively worsening bilateral proptosis presented to our hospital. She noticed bilateral proptosis 5 years ago. Although her thyroid tests were normal, she underwent retrobulbar injection of triamcinolone acetonide with no improvement of symptoms. She was diagnosed as having new onset of diabetes and hypertension during this time and was treated with insulin and amlodipine, respectively. Later she started developing proximal muscle weakness and was easily bruised respectively. She was a farmer belonging to a middle class family, living with her husband and two children, all healthy, in a remote city where she had lived for a major part of her life. She did not smoke tobacco or consume alcohol. She had no significant past medical history. She had not had any chronic illness or infectious disease previously. There was no family history of similar disease or any other chronic illness such as diabetes and hypertension. There was no history of allergy to any food or drugs. On physical examination: temperature $(\mathrm{T}) 36.5^{\circ} \mathrm{C}$, pulse $(\mathrm{P}) 72$ beats/minute, respiration rate (R) 20 breaths/minute, blood pressure (BP) 140/90 mmHg, weight (W) $60 \mathrm{~kg}$, and height (H) 156 $\mathrm{cm}$. She showed typical Cushingoid appearance: skin atrophy, moon facies, buffalo hump, and purplish abdominal striae. She also had conjunctival congestion, edema, and lid retraction. Her respiratory movements were bilaterally symmetrical with a frequency of 20 breaths/minute. A cardiac examination was normal. S1 and S2 were normal with no added sounds. On abdominal examination no masses or tenderness were noted on both light and deep palpation. Her liver and spleen were not palpable. A sensory and motor system examination did not reveal any abnormality. Her neurological reflexes were normal. All cranial nerves were intact. Further ophthalmological examination revealed bilateral proptosis with Hertel's exophthalmometer readings of $21 \mathrm{~mm}$ (right) and $22 \mathrm{~mm}$ (left). Intraocular pressure was elevated in her right eye $(26 \mathrm{mmHg})$ and her left eye $(27$ $\mathrm{mmHg}$ ). A fundus examination was normal. Extraocular movements were intact and bilateral visual fields showed no defect.
Complete blood count, urine analysis, and renal and liver function tests were all normal. A biochemical evaluation revealed elevated 24-hour urinary free cortisol (UFC) $306.8 \mu \mathrm{g} / 24$ hours (reference range 3.5 to $45 \mu \mathrm{g} /$ 24 hours), elevated cortisol level, and suppressed ACTH level with loss of their normal diurnal rhythm. Her cortisol level was $634 \mathrm{ug} / \mathrm{L}$ at 8 a.m., $621 \mathrm{ug} / \mathrm{L}$ at 4 p.m., and $521 \mathrm{ug} / \mathrm{L}$ at $0 \mathrm{a.m}$. (reference range 62 to $194 \mathrm{ug} / \mathrm{L}$ ), while her ACTH level was always $<1 \mathrm{ng} / \mathrm{L}$ (reference range 7.2 to $63.6 \mathrm{ng} / \mathrm{L}$ ). Serum cortisol remained unsuppressed after $1 \mathrm{mg}$ overnight and high-dose dexamethasone suppression tests (at $624 \mathrm{ug} / \mathrm{L}$ and $607 \mathrm{ug} / \mathrm{L}$, respectively). Serum thyroid function tests were normal; anti-thyrotropin receptor antibody (TRAb), anti-thyroid peroxidase (TPOAb), and anti-thyroglobulin antibodies (TGAb) were negative. Plasma potassium was 2.2 3.0 $\mathrm{mmol} / \mathrm{L}$. Glycated hemoglobin (HbA1c) was $8.7 \%$. Serum androgen, aldosterone/renin activity ratio, and 24-hour urinary catecholamines were all in the normal range. Glucagon, luteinizing hormone-releasing hormone (LHRH), mixed meal, postural, metoclopramide, and vasopressin tests were performed in order to evaluate aberrant hormonal responses. Results were positive for vasopressin and upright posture test. Computed tomography $(\mathrm{CT})$ imaging of her adrenal glands revealed bilateral multiple lobular masses (Fig. 1a, b); magnetic resonance imaging (MRI) of her orbits indicated bilateral exophthalmos with hypertrophy of the retro-orbital fat (Fig. 1c); MRI of her pituitary was normal (Fig. 1d). Diagnosis of PBMAH was made and our patient underwent bilateral laparoscopic adrenalectomy. Gross pathological examination of adrenal glands showed each gland contained several nodules (Fig. 1e). Pathological findings were consistent with PBMAH: multinodular glands with homogenous, golden-yellow-colored nodules. Nodules contained predominantly fascicular cells (Fig. 1f). After surgery, her plasma cortisol and ACTH at 8 a.m. were $6.06 \mathrm{ug} / \mathrm{L}$ and $1.87 \mathrm{ng} / \mathrm{L}$ respectively, indicating a successful surgery. Hydrocortisone supplementation was given as well, as she was started on metformin for diabetes. At 6-month follow-up, her blood glucose levels were well controlled and her blood pressure was 120/80 $\mathrm{mmHg}$ without use of any antihypertensive medication. The exophthalmos improved markedly after the surgery.

After obtaining informed consent from our patient, DNA was extracted from peripheral blood leukocytes and seven different adrenal nodules that were obtained during surgery. All the coding and flanking intronic sequences of $A R M C 5$ were amplified by polymerase chain reaction (PCR). Mutations were identified by direct sequencing of PCR products. For the variations nomenclature, the main frequent isoform in the literature (NM_001105247.1) was used. We identified a heterozygous nonsense $A R M C 5$ germline mutation c.682C $>\mathrm{T}$ (p. 

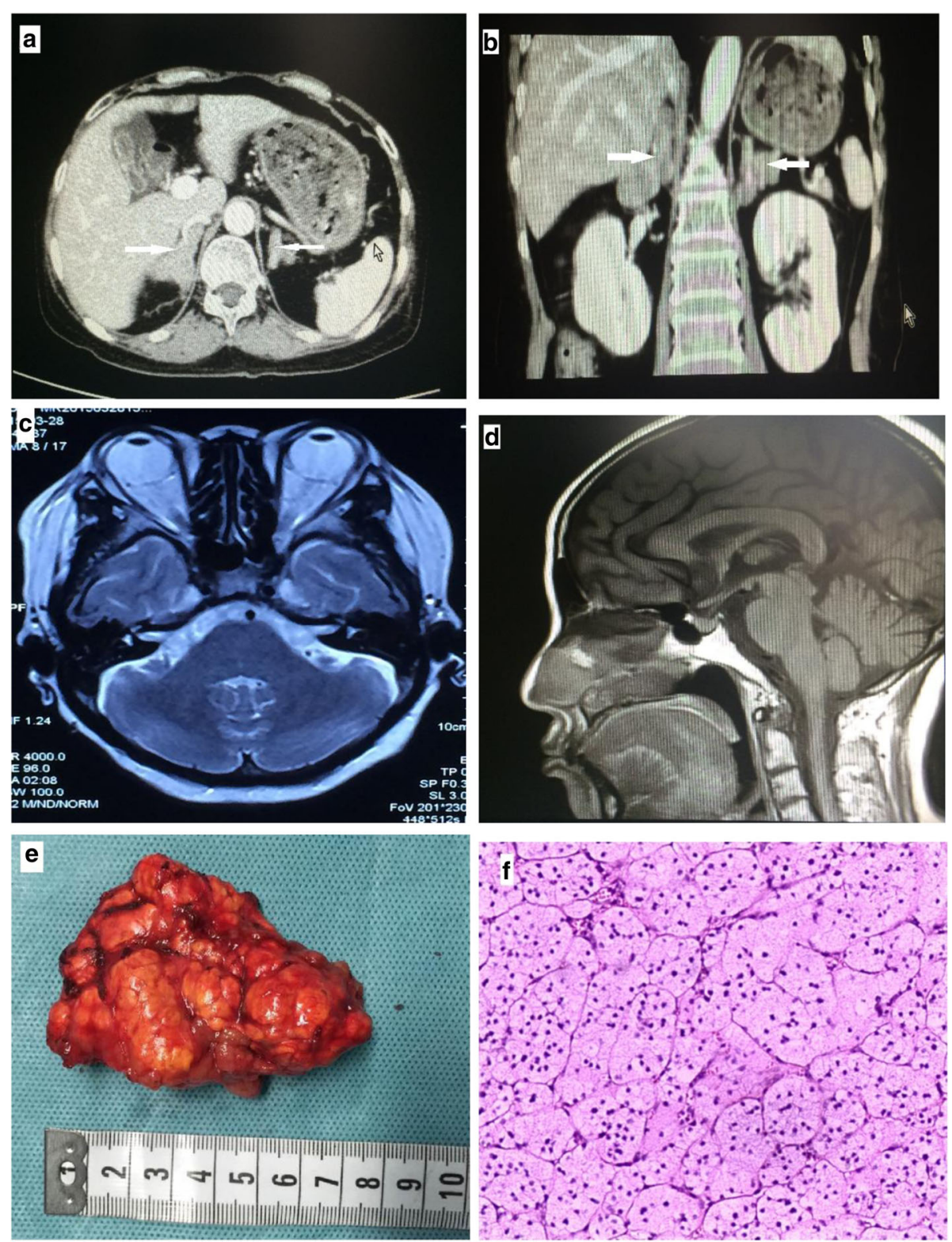

Fig. 1 Clinical features of the patient with primary bilateral macronodular adrenal hyperplasia. Computed tomography scan of adrenal glands showed massive enlargement of the adrenal glands and multiple nodules (as shown by arrows) (a, $\mathbf{b}$ ). Magnetic resonance imaging of the orbital showed bilateral exophthalmos (c). MRI of the pituitary gland was normal (d).Gross pathology of the resected left adrenal gland with nodules of various sizes (e). Histological view of resected adrenal tissue showing the presence of large cortical cells shown by hematoxylin and eosin stain at magnification $\times 100(\mathbf{f})$

Gln228*) in exon 3, which was predicted to change amino acid glutamine at position 228 to a stop codon (Fig. 2a). We also identified five heterozygous frameshift mutations (c.310delG, c.347_357del11, c.267delC, c.283 289del7, and c.205-322del118) in five different adrenal nodules (Fig. 2b-f). These mutations are predicted to cause a downstream stop codon with premature termination of translation (p.Ala104Profs*33, p.Ser116Tyrfs*6, p.Pro89Profs*48, p.Ser95Argfs*40, and
p.Pro69Alafs*29 respectively). We did not find $A R M C 5$ mutations in the remaining two adrenal nodules. All the mutations are novel and not found in available online databases. Screening in first-degree relatives of our patient revealed no carriers of an ARMC5 mutation.

Total ribonucleic acid (RNA) from the adrenal tumor tissue and two specimens of normal adrenal cortex were extracted and were reverse transcribed using a cDNA synthesis kit. To test whether the ARMC5 mutation 


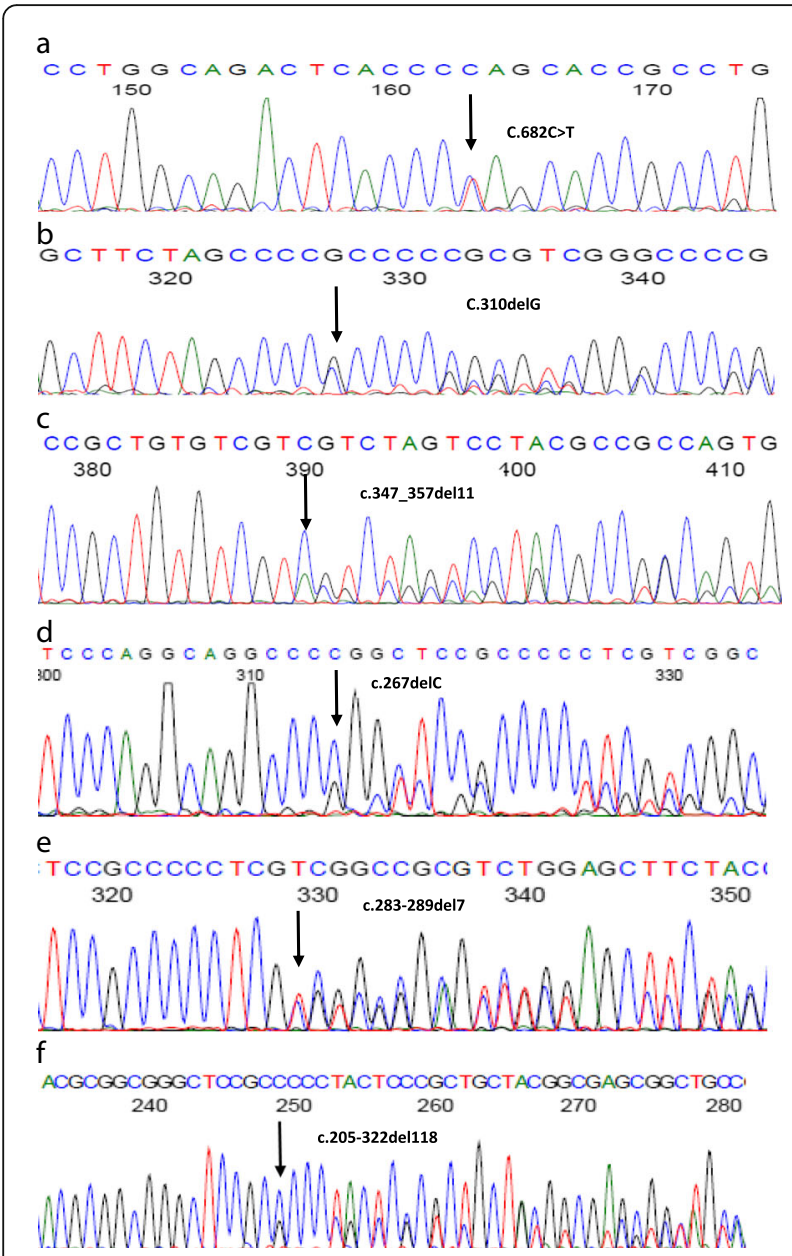

Fig. 2 Germline and five somatic mutations in the ARMC5 gene found in the patient with primary bilateral macronodular adrenal hyperplasia. Heterozygous germline mutation C.682C>T was present in all analyzed tissue (a). Heterozygous somatic mutations were found in five different nodules: C.310delG (b), c.347_357del11 (c), c.267delC (arrow) (d), c.283-289del7 (e), and c.205-322del118 (f)

induced messenger RNA (mRNA) decay, real-time reverse transcriptase (RT)-PCR was performed. The $A R M C 5$ cDNA sequence was amplified with specific primers (5'-CTGGAGTGCAGTGACACGAT-3' and 5' TATCTGGGCATGGTGGTACA-3'). The relative ARM C5 mRNA expression was determined by using the 2 $-\triangle \Delta C T$ method and was normalized against the expression of the $\beta$-actin gene. The qRT-PCR analysis indicated the ARMC5 mRNA expression was lower in our patient's adrenal tumor samples compared to normal adrenal cortex (Fig. 3).

\section{Discussion}

In our study, we present a case of a 52-year-old woman with a history of long-standing exophthalmos which was thought to be Graves' ophthalmopathy but persisted despite treatment and she was later diagnosed as having

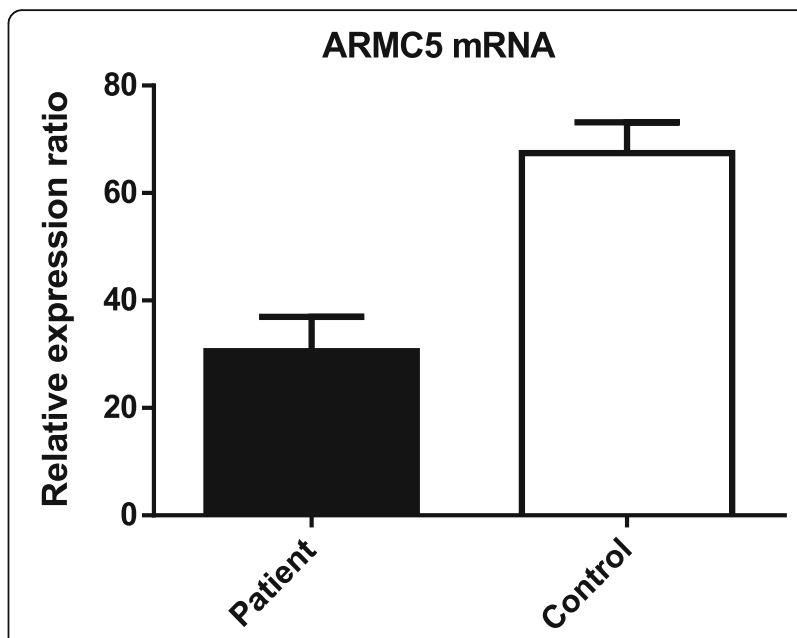

Fig. 3 The messenger ribonucleic acid expression of ARMC5 in the adrenal tumor samples of the patient and normal adrenal cortex. The ARMC5 messenger RNA expression was lower in patient's adrenal tumor samples compared to normal adrenal cortex. Patient is represented by black bar and control sample by white bar

Cushing's syndrome due to primary bilateral macronodular hyperplasia. There have been only a few reports on atypical symptoms of Cushing's syndrome and this report highlights the importance of exophthalmos and its association with hypercortisolism.

PBMAH is responsible for less than $1 \%$ of endogenous Cushing's syndrome; quite often it has been found incidentally [3]. These tumors usually have low secretory capacity and result in subclinical Cushing's syndrome over an extended period of time, leading to delay in diagnosis in a majority of patients. Although exophthalmos is typically associated with Graves' disease, it has also been described in Cushing's syndrome. As estimated [9], exophthalmos was present in $45 \%$ of active Cushing's syndrome, $21 \%$ of iatrogenic Cushing's syndrome, and $20 \%$ of treated Cushing's syndrome in comparison to $2 \%$ in controls. The exact mechanism behind exophthalmos remains to be identified. It has been proposed that this is part of generalized fat redistribution typically seen in hypercortisolism. The excessive fat deposition in the orbit spares the orbital muscles. Some suggest increased lipoprotein lipase activity or increased glucocorticoid receptor density may be the cause behind this phenomenon [10-12]. Exophthalmos is an often forgotten clinical sign of Cushing's syndrome at this time. In our study, we presented a case of PBMAH who only had the exophthalmos as a major symptom at the beginning and was treated as Graves' ophthalmopathy until the classic features of Cushing's syndrome appeared. This highlights the importance of early recognition of atypical symptoms of Cushing's syndrome such as exophthalmos, which would save the patient from harmful effects of excessive cortisol exposure 
for an extended period of time and may help prevent the complications as well.

Recently, inactivating ARMC5 mutations have been identified as a common genetic cause of PBMAH [3]. In our cohort, we identified a novel germline mutation c. $682 \mathrm{C}>\mathrm{T}$ and five novel somatic mutations in five different adrenocortical nodules which was in agreement with previous studies that most of the mutations observed were private and only a minority of them recurred [13, 14]. Although rearrangements or larger genomic deletion cannot be excluded in the remaining two nodules, the finding indicated that not every nodule carried another ARMC5 variant [14]. Thus far, more than 30 germline and somatic pathogenic mutations have been reported $[3-8,14,15]$. Nonsense and frameshift mutations leading to nonsense transcripts contribute to nearly $60 \%$ of all pathogenic variants. It is known that mutations introducing premature translation stop codons (PTCs) trigger the mRNA surveillance pathway and nonsense-mediated decay [16]. In our study, the nonsense germline mutation and five frameshift somatic mutations all resulted in a premature stop codon and the corresponding mRNA could thus possibly be subjected to degradation through the nonsense-mediated decay. This hypothesis was supported by the fact that the total ARMC5 mRNA expression was relatively low in our patient compared to control adrenal cortex. The exact function of ARMC5 protein and its associated signaling pathway are still largely unknown. The in vitro studies showed that transfecting the wild-type ARMC5 gene induced apoptosis, while introducing ARMC5 missense mutations abolished this effect [3, 17]. Thus, $A R M C 5$ inactivation leads to resistance to apoptosis in adrenocortical cells, which causes adrenal hyperplasia leading to increased cortisol level. Phenotype-genotype relations studies showed that patients with the ARMC5damaging mutation exhibited a more severe hypercortisolism and larger adrenals compared to patients with the wild-type [13, 17]. Some reports also showed that $A R M C 5$ mutations are associated with specific patterns of illegitimate receptors expression. In our study, we noted abnormal responses after upright posture and vasopressin administration, which is in agreement with previous observations $[3,18]$.

\section{Conclusions}

We demonstrated extensive genetic diversity of $A R M C 5$ in a patient with PBMAH that started with exophthalmos, which further expands the molecular pathophysiology of this disease. Early recognition of atypical symptoms and screening for ARMC5 mutation in patients with PBMAH has important clinical implications for the diagnosis and genetic counseling.

\section{Abbreviations}

ACTH: Adrenocorticotropic hormone; AIMAH: ACTH-independent macronodular adrenal hyperplasia; ARMC5: Armadillo repeat containing 5; BP: Blood pressure; CT: Computed tomography; H: Height; HbA1c: Glycated hemoglobin; LHRH: Luteinizing hormone-releasing hormone; mRNA: Messenger RNA; P: Pulse; PBMAH: Primary bilateral macronodular adrenal hyperplasia; PCR: Polymerase chain reaction; PTC: Premature translation stop codon; R: Respiration rate; RT: Reverse transcriptase; T: Temperature; TGAb: Anti-thyroglobulin antibodies; TPOAb: Anti-thyroid peroxidase; TRAb: Anti-thyrotropin receptor antibody; UFC: Urinary free cortisol; W: Weight

\section{Acknowledgements}

We wish to thank the patient and her relatives for consenting to the publication of this case report.

\section{Funding}

This study was supported by the National Natural Science Foundation of China $(81670730,81100583)$, Natural Science Foundation of Hunan Province (2016JJ4103, 2015JJ4095).

\section{Availability of data and materials}

All laboratory data relevant to this case report can be found in the "Case presentation" section of this report.

\section{Authors' contributions}

$\mathrm{PJ}$ is the first author and wrote this manuscript; MUJ and QZ performed the research. CSD and YY coordinated the research; PJ and ZHM designed the research study. All authors read and approved the final manuscript.

\section{Ethics approval and consent to participate}

All procedures followed were in accordance with the ethical standards of the responsible committee on human experimentation (institutional and national) and with the Declaration of Helsinki of 1975, as revised in 2000.

\section{Consent for publication}

Written informed consent was obtained from the patient for publication of this case report and any accompanying images. A copy of the written consent is available for review by the Editor-in-Chief of this journal.

\section{Competing interests}

The authors declare that they have no competing interests.

\section{Publisher's Note}

Springer Nature remains neutral with regard to jurisdictional claims in published maps and institutional affiliations.

\section{Author details}

${ }^{1}$ Department of Endocrinology, The third Xiangya Hospital, Central South University, Tongzipo Road, Changsha 410007, Hunan, People's Republic of China. ${ }^{2}$ Department of Anesthesia, The affiliated Tumor Hospital of Xiangya Medical School of Central South University, Changsha 410007, Hunan, China.

Received: 9 October 2017 Accepted: 27 November 2017

Published online: 18 January 2018

\section{References}

1. Lacroix A. ACTH-independent macronodular adrenal hyperplasia. Best Pract Res Clin Endocrinol Metab. 2009;23(2):245-59.

2. Louiset E, Duparc C, Young J, Renouf S, Tetsi Nomigni M, Boutelet I, et al. Intraadrenal corticotropin in bilateral macronodular adrenal hyperplasia. N Engl J Med. 2013;369:2115-25.

3. Assié G, Libé R, Espiard S, Rizk-Rabin M, Guimier A, Luscap W, et al. ARMC5 mutations in macronodular adrenal hyperplasia with Cushing's syndrome. N Engl J Med. 2013;369:2105-14.

4. Alencar GA, Lerario AM, Nishi MY, Mariani BM, Almeida MQ, Tremblay J, et al. ARMC5 mutations are a frequent cause of primary macronodular adrenal Hyperplasia. J Clin Endocrinol Metab. 2014;99(8):E1501-9.

5. Faucz FR, Zilbermint M, Lodish MB, Szarek E, Trivellin G, Sinaii N, et al. Macronodular adrenal hyperplasia due to mutations in an armadillo repeat 
containing 5 (ARMC5) gene: a clinical and genetic investigation. J Clin Endocrinol Metab. 2014;99:E1113-9.

6. Albiger NM, Regazzo D, Rubin B, Ferrara AM, Rizzati S, Taschin E, et al. A multicenter experience on the prevalence of ARMC5 mutations in patients with primary bilateral macronodular adrenal hyperplasia: from genetic characterization to clinical phenotype. Endocrine. 2017;55(3):959-68.

7. Gagliardi L, Schreiber AW, Hahn CN, Feng J, Cranston T, Boon H, et al. ARMC5 Mutations are common in familial bilateral macronodular adrenal hyperplasia. J Clin Endocrinol Metab. 2014;99(9):E1784-92.

8. Elbelt U, Trovato A, Kloth M, Gentz E, Finke R, Spranger J, et al. Molecular and clinical evidence for an ARMC5 tumor syndrome: concurrent inactivating germline and somatic mutations are associated with both primary macronodular adrenal hyperplasia and meningioma. J Clin Endocrinol Metab. 2015;100(1):E119-28.

9. Kelly W. Exophthalmos in Cushing's syndrome. Clin Endocrinol. 1996:45(2): $167-70$.

10. Panzer SW, Patrinely JR, Wilson HK. Exophthalmos and iatrogenic Cushing's syndrome. Ophthal Plast Reconstr Surg. 1994;10(4):278-82.

11. Peyster RG, Ginsberg F, Silber JH, Adler LP. Exophthalmos caused by excessive fat: CT volumetric analysis and differential diagnosis. AJR Am J Roentgenol. 1986;146(3):459-64.

12. Giugni AS, Mani S, Kannan S, Hatipoglu B. Exophthalmos: A Forgotten Clinical Sign of Cushing's Syndrome. Case Rep Endocrinol. 2013;2013: 205208.

13. Lacroix A. Heredity and cortisol regulation in bilateral macronodular adrenal hyperplasia. N Engl J Med. 2013;369(22):2147-9.

14. Correa R, Zilbermint M, Berthon A, Espiard S, Batsis M, Papadakis GZ, et al. The ARMC5 gene shows extensive genetic variance in primary macronodular adrenocortical hyperplasia. Eur J Endocrinol. 2015;173(4):435-40.

15. Zilbermint M, Xekouki P, Faucz FR, Berthon A, Gkourogianni A, Schernthaner-Reiter MH, et al. Primary aldosteronism and ARMC5 variants. $J$ Clin Endocrinol Metab. 2015;100(6):E900-9.

16. Khajavi M, Inoue K, Lupski JR. Nonsense-mediated mRNA decay modulates clinical outcome of genetic disease. Eur J Hum Genet. 2006;14:1074-81.

17. Espiard S, Drougat L, Libé R, Assié G, Perlemoine K, Guignat L, Barrande G, Brucker-Davis $F$, et al. ARMC5 mutations in a large cohort of primary macronodular adrenal hyperplasia: clinical and functional consequences. J Clin Endocrinol Metab. 2015;100(6):E926-35.

18. Libé R, Coste J, Guignat L, Tissier F, Lefebvre H, Barrande G, Ajzenberg C, et al Aberrant cortisol regulations in bilateral macronodular adrenal hyperplasia: a frequent finding in a prospective study of 32 patients with overt or subclinical Cushing's syndrome. Eur J Endocrinol. 2010;163(1):129-38.

\section{Submit your next manuscript to BioMed Central and we will help you at every step:}

- We accept pre-submission inquiries

- Our selector tool helps you to find the most relevant journal

- We provide round the clock customer support

- Convenient online submission

- Thorough peer review

- Inclusion in PubMed and all major indexing services

- Maximum visibility for your research

Submit your manuscript at www.biomedcentral.com/submit
Biomed Central 